\title{
Quantum Teleportation of Light
}

\author{
Changsuk Noh ${ }^{\dagger}$, A. Chia ${ }^{\S}$, Hyunchul Nha ${ }^{\ddagger}$, M. J. Collett ${ }^{\dagger}$, and H. J. Carmichael ${ }^{\dagger}$ \\ ${ }^{\dagger}$ Department of Physics, University of Auckland, Private Bag 92019, Auckland, New Zealand \\ ${ }^{\S}$ Centre for Quantum Dynamics, Griffith University, Nathan, Queensland 4111, Australia \\ ${ }^{\ddagger}$ Department of Physics, Texas A \&3 M University at Qatar, Dohar, Qatar
}

(Dated: November 5, 2018)

\begin{abstract}
Requirements for the successful teleportation of a beam of light, including its temporal correlations, are discussed. Explicit expressions for the degrees of first- and second-order optical coherence are derived. Teleportation of an antibunched photon stream illustrates our results.

PACS numbers: 03.65.Ud, 03.67.-a, 42.50.-p
\end{abstract}

Although proposed originally by Vaidman [1] as a protocol for teleporting the wavefunction of a particle, continuous-variable teleportation has primarily been developed as a scheme for teleporting the quantum state of light. Braunstein and Kimble 2] recognized how Vaidman's idea might be implemented to teleport the state of a single mode of the electromagnetic field employing squeezed-state entanglement. Subsequent experiments have largely followed their single-mode prescription [3, 4, 5], although these experiments are inherently broadband and suggest a generalization from the teleportation of quantum states to the teleportation of quantum fields.

A broadband theory was developed by van Loock et al. [6] and teleportation of broadband entanglement has recently been reported [7]. Neither of these works, however, fully explores the implications of the generalization to quantum fields. It is our aim in this Letter to develop that generalization. Specifically, we propose teleporting a quantum field (beam of light) in the following sense: as a consequence of Alice's measurements and Bob's actions, a light beam emerges at the teleporter output carrying over all the statistical properties of the beam at the input; in this the quantum field is characterized by correlation functions in time - ideally to all orders - rather than by a single-mode quantum state.

Naively one might expect this to be achieved so long as the squeezing bandwidth is larger than the bandwidth of the input light. This, however, is not the case, as vacuum modes are equally a part of a quantum field; for the purposes of teleportation as proposed, no quantum field may be viewed as having a bandwidth less than the squeezing. What is required, then, is optical filtering by Bob: we propose that the vacuum lying outside the input light bandwidth not be teleported, but rather be replaced by an equivalent vacuum reflected by Bob's filter.

Experiments to date take a much different view. They (as Victor [3]) measure properties of the teleported light over a narrow span of frequencies only, i.e., they filter in detection. Also, they measure only quadrature phase amplitudes. Thus, they leave aside temporal correlations and, in particular, correlations to be described by operators in different orders. Our aim is to expand upon their point of view: we ask that any conceivable measurement at the input yield equivalent results at the output; then one can say the light (quantum field) is teleported.

Any conceivable measurement is a tall order when the infinite dimensions of a field are considered. In this communication we focus on photon correlations, specifically a measurement of $g^{(2)}(\tau)$. We ask whether and under what conditions an antibunched photon stream at the input will appear, with high fidelity, as an antibunched photon stream at the output. The anticorrelation of photon arrival times places high demands on the reproduction of the input quantum field across its entire bandwidth, including all vacuum modes. Moreover, as an expression of the particle aspect of light, photon correlations play as counterpoint to the wave amplitude measurements upon which continuous variable teleportation and its characterization in experiments to date are based; they require attention be given to the issue of operator order.

We begin by outlining our model of the standard continuous variable teleporter [3]. We work in the time domain and for the purpose of setting notation, start with all fields, including vacuum fields, represented by classical stochastic processes. These are Wigner stochastic processes in conventional terms, where, for the time being, positive-definite Wigner functions are assumed; they also conform to the viewpoint of stochastic electrodynamics [8]. A sketch of the model appears in Fig. [1. In units of photon flux, input and squeezed fields are written as

$$
\begin{aligned}
\mathcal{E}_{\mathrm{in}} & =\sqrt{2 \gamma_{i}} c-\xi_{\mathrm{in}}^{t}, \\
X_{\mathrm{sq}} & =\sqrt{2 \gamma_{s}} a-\xi_{a}^{t}, \\
Y_{\mathrm{sq}} & =\sqrt{2 \gamma_{s}} b-\xi_{b}^{t},
\end{aligned}
$$

where $2 \gamma_{i}$ and $2 \gamma_{s}$ are field bandwidths, and $\xi_{\text {in }}^{t}, \xi_{a}^{t}, \xi_{b}^{t}$ are Gaussian while noises, i.e., vacuum fields. Alice measures the complex valued photocurrent $I=I_{X}+i I_{Y}$, with

$$
d I / d t=-\gamma_{A}(I-d Q / d t)
$$

where $2 \gamma_{A}$ is her measurement bandwidth, and

$$
\frac{d Q}{d t}=\frac{1}{\sqrt{2}} \mathcal{E}_{\text {in }}+\frac{1}{2}\left(X_{\mathrm{sq}}+Y_{\mathrm{sq}}\right)^{*}
$$




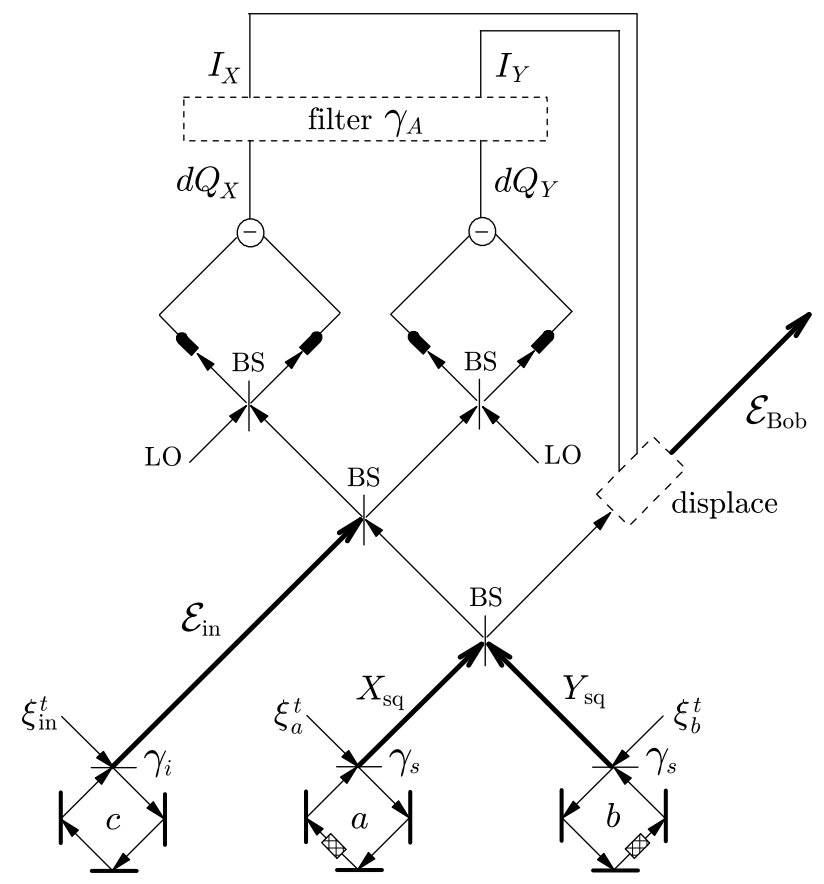

FIG. 1: Schematic of continuous variable teleportation with no filtering of the output by Bob; BS: 50/50 beam splitter; LO: local oscillator.

Bob implements the displacement $\sqrt{2} I$ to produce

$$
\begin{aligned}
\mathcal{E}_{\mathrm{Bob}}= & \frac{1}{\sqrt{2}}\left(X_{\mathrm{sq}}-Y_{\mathrm{sq}}\right) \\
& +F_{A} *\left[\mathcal{E}_{\mathrm{in}}+\frac{1}{\sqrt{2}}\left(X_{\mathrm{sq}}+Y_{\mathrm{sq}}\right)^{*}\right],
\end{aligned}
$$

where $F_{A}(t)$ is the impulse response of Alice's filter and * denotes convolution. If we then assume a broad measurement bandwidth for Alice, $\gamma_{A} \gg \gamma_{s}, \gamma_{i}$, approximating $F_{A}(t)$ as a $\delta$-function yields

$$
\mathcal{E}_{\mathrm{Bob}}=\mathcal{E}_{\mathrm{in}}+\sqrt{2}\left(X_{\mathrm{sq}}^{X}-i Y_{\mathrm{sq}}^{Y}\right) .
$$

It seems that perfect squeezing, $X_{\mathrm{sq}}^{X}=Y_{\mathrm{sq}}^{Y}=0$, leads to perfect teleportation, $\mathcal{E}_{\mathrm{Bob}}=\mathcal{E}_{\text {in }}$. We must note the assumption $\gamma_{A} \gg \gamma_{s}$, however, since according to it the squeezing cannot be perfect across Alice's entire bandwidth. Nevertheless, the ideal result can be recovered if $\gamma_{s}=\gamma_{A}$ and both Alice's spectrum and the squeezing spectrum are flat. Then, for perfect squeezing,

$$
\mathcal{E}_{\mathrm{Bob}}=\left\{\begin{array}{cl}
\mathcal{E}_{\text {in }}, & |\omega|<\gamma_{s}=\gamma_{A} \\
\left(\xi_{a}^{t}-\xi_{b}^{t}\right) / \sqrt{2}, & |\omega|>\gamma_{s}=\gamma_{A},
\end{array}\right.
$$

where here and in what follows $\omega$ is measured relative to the input light line center. Outside the squeezing bandwidth, Eq. (6) simply replaces $X_{\mathrm{sq}}$ and $Y_{\mathrm{sq}}$ [first term on the right in Eq. (4)] by the vacuum fields $\xi_{a}^{t}$ and $\xi_{b}^{t}$. The result, being a vacuum field, is operationally equivalent to teleporting $\xi_{\text {in }}^{t}$.
This matched-spectra scenario is highly idealized, though, and it is more realistic to take $\gamma_{A}>\gamma_{s}$ and allow both spectra a frequency roll off; Alice's bandwidth must of course exceed the squeezing bandwidth, otherwise it is not possible to completely cancel the squeezed-light noise distributed to Bob. Under these conditions, Bob might then filter his field (Fig. 2), to generate the output

$$
\mathcal{E}_{\text {out }}=F_{B} *\left(\mathcal{E}_{\text {Bob }}+\xi_{\text {out }}^{t}\right)-\xi_{\text {out }}^{t},
$$

with $F_{B}(t)$ the impulse response of Bob's filter; the filter bandwidth is $2 \gamma_{B}$ and $\xi_{\text {out }}^{t}$ is the vacuum reflected at its output.

We now ask under what conditions does $\mathcal{E}_{\text {out }}=\mathcal{E}_{\text {in }}$ or $\mathcal{E}_{\text {out }}=\xi_{\text {out }}^{t},|\omega| \gg \gamma_{i}$, and does such perfect teleportation carry over to the teleportation of an antibunched photon stream? To be more explicit, we might set $\mathcal{E}_{\text {in }}$ as the scattered field from a driven two-state atom (resonance fluorescence). What then are the requirements on the employed squeezing and relative bandwidths such that Bob's filter (mode $d$ of Fig. 2) behaves as a two-state system, i.e., is never occupied by more than one photon?

In addressing these questions the classical (Wigner) representation of stochastic processes must be dropped. Strictly, only $I$ and $Q$ in the above equations are cnumbers; all the fields are operators. If, then, one aims to realize Alice's classical information, an explicit modeling of her measurements is needed to connect the operators to the c-numbers. Quantum trajectory theory does this with

$$
\frac{d Q}{d t}=\frac{1}{\sqrt{2}}\left\langle\hat{\mathcal{E}}_{\text {in }}\right\rangle_{\text {rec }}+\frac{1}{2}\left(\left\langle\hat{X}_{\mathrm{sq}}^{\dagger}\right\rangle_{\text {rec }}+\left\langle\hat{Y}_{\mathrm{sq}}^{\dagger}\right\rangle_{\text {rec }}\right)-\xi_{\text {shot }}^{t}
$$

in place of Eq. (3). Note the shot noise, $\xi_{\text {shot }}^{t}$, in place of the classical representation of vacuum fluctuations, $\xi_{\text {in }}^{t} / \sqrt{2}+\left(\xi_{a}^{t *}+\xi_{b}^{t *}\right) / 2$. The difficulty with this approach is that although low fidelity teleportation can be simulated [9] , numerical simulation of high-fidelity conditions is impractical. In response we adopt an alternative method of calculation that while it does not realize Alice's classical

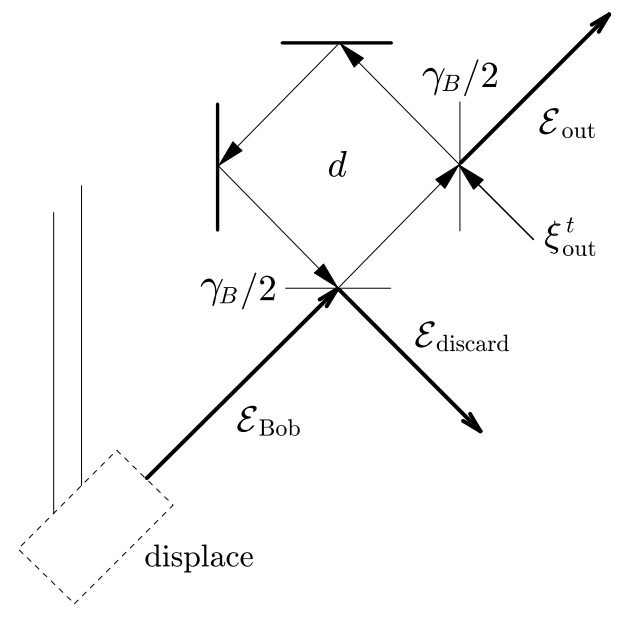

FIG. 2: Schematic of Bob's filter. 
information, does provide analytical expressions for correlation functions - for any operator order. We replace the classical fields $\mathcal{E}_{\text {in }}, X_{\mathrm{sq}}, Y_{\mathrm{sq}}$, etc. by operators, and the c-numbers $I$ and $Q$ by formal operators [10]. Then the same linear map from input to output holds as an operator expression. Output field correlation functions may be determined from the known correlation functions of $\hat{\mathcal{E}}_{\text {in }}, \hat{X}_{\mathrm{sq}}$, and $\hat{Y}_{\mathrm{sq}}$.

We have carried this program through to derive general expressions, functions of all parameters, for the degrees of first and second-order optical coherence. While these expressions are used to generate the plots of Figs. 3 and 4. they are too unwieldy to reproduce in general form. We present them simplified for large relative bandwidths, $\gamma_{A} \gg \gamma_{B}, \gamma_{s}, \gamma_{i}$, and $\gamma_{B} \gg \gamma_{i}$. Then if $g_{\text {in }}^{(1)}(\tau)$ and $g_{\text {in }}^{(2)}(\tau)$ are the input field degrees of first- and second-order optical coherence, we find for the teleported light:

$$
f_{\text {out }} g_{\text {out }}^{(1)}(\tau)=f_{s} A_{s}(\tau)+f_{\text {in }} g_{\text {in }}^{(1)}(\tau),
$$

where $f_{\text {in }}=\left\langle\hat{\mathcal{E}}_{\text {in }}^{\dagger} \hat{\mathcal{E}}_{\text {in }}\right\rangle$ and $f_{\text {out }}=\left\langle\hat{\mathcal{E}}_{\text {out }}^{\dagger} \hat{\mathcal{E}}_{\text {out }}\right\rangle$ are input and output field photon fluxes, and

$$
\frac{f_{s}}{\gamma_{B}} A_{s}(\tau)=\frac{\frac{1}{2}\left(\gamma_{B}^{2}-\gamma_{-}^{2}\right) e^{-\gamma_{B} \tau}-\frac{2 \lambda}{1+\lambda} \gamma_{B} \gamma_{s} e^{-\gamma_{+} \tau}}{\gamma_{B}^{2}-\gamma_{+}^{2}},
$$

with $A_{s}(0) \equiv 1$ and $\gamma_{ \pm} \equiv \gamma_{s}(1 \pm \lambda)$; and

$$
\begin{aligned}
g_{\text {out }}^{(2)}(\tau)= & 1+\left(\frac{f_{\text {in }}}{f_{\text {out }}}\right)^{2}\left[g_{\text {in }}^{(2)}(\tau)-1\right] \\
& +\frac{f_{s}}{f_{\text {out }}} A_{s}(\tau)\left[g_{\text {out }}^{(1)}(\tau)+\frac{f_{\text {in }}}{f_{\text {out }}} g_{\text {in }}^{(1)}(\tau)\right] .
\end{aligned}
$$

The parameter $\lambda$ determines the degree of line-center squeezing at the output of a sub-threshold parametric oscillator, which is given in $\mathrm{dB}$ by $-20 \log [(1-\lambda) /(1+\lambda)]$.

The spectrum of the teleporter output light is the Fourier transform of $g_{\text {out }}^{(1)}(\tau)$. We discuss it first to illustrate the interplay of the various bandwidths, $\gamma_{A}, \gamma_{B}$, $\gamma_{i}$, and $\gamma_{s}$. We consider the example of input resonance fluorescence, i.e., teleportation of the Mollow triplet. Results are displayed in Fig. 3, where the incoherent part of the spectrum, $s_{\text {out }}(\omega)$, is plotted as a function of Rabi frequency $\Omega$. Quite generally, from Eq. (91), the output spectrum is the spectrum of the input light sitting on a noise background. The relative size of the pieces (integrated over $\omega$ ) is

$$
\frac{f_{s}}{f_{\text {in }}}=\frac{\gamma_{B}}{\eta \gamma_{i}}\left(\frac{1}{2}-\frac{2 \lambda}{1+\lambda} \frac{1}{1+\lambda+\gamma_{B} / \gamma_{s}}\right) \frac{\Omega^{2}+2 \gamma_{i}^{2}}{\Omega^{2}},
$$

from Eq. (10) and the photon flux directed to the teleporter input, $f_{\text {in }}=\eta \gamma_{i} \Omega /\left(\Omega^{2}+2 \gamma_{i}^{2}\right) ; 2 \gamma_{i}$ is the Einstein $A$ coefficient and $\eta<1$ is a collection efficiency. For $\lambda=0$ (classical teleportation), the factor $1 / 2$ inside the bracket is the mean number of photons, $\left\langle\hat{d}^{\dagger} \hat{d}\right\rangle=1 / 2$, in Bob's filter. It may be traced, formally, to the vacuum fluctuations presented to Alice - a product of the conjugate on the dispersed-field contribution, $\left(\hat{X}_{\mathrm{sq}}^{\dagger}+\hat{Y}_{\mathrm{sq}}^{\dagger}\right) / \sqrt{2}$, in the formal operator representation of Alice's photocurrent [Eqs. (3) and (4)]; physically, however, it is a number of real photons generated through Bob's displacement by Alice's shot noise - $\xi_{\text {shot }}^{t}$ in Eq. (8) [no photons travel to Bob via the dispersed field $\left(\hat{X}_{\mathrm{sq}}-\hat{Y}_{\mathrm{sq}}\right) / \sqrt{2}$ ].

The aim in quantum teleportation is to remove the background. Figure 3 illustrate how this might be done. In frame (a), for any $\lambda>0$, the background is reduced over the squeezing bandwidth. Here the displacement driven by Alice's photocurrent partially nulls the light now dispersed to Bob; but a residual background persists across Alice's detector bandwidth (with $\gamma_{B}>\gamma_{A}$ ). In frames (b) and (c) filtering $\left(\gamma_{B}<\gamma_{A}\right)$ suppresses it near line center, although not fully in the wings. In frame (d), with $\gamma_{A} \gg \gamma_{s} \gg \gamma_{B} \gg \gamma_{i}$ and sufficient squeezing, near perfect teleportation of the Mollow triplet is achieved; note, for example, that the bracket in Eq. (12) goes to zero for $\gamma_{B} / \gamma_{s} \rightarrow 0$ and $\lambda \rightarrow 1$.

Turning now to the teleportation of an antibunched photon stream, $f_{s} / f_{\text {in }}$ is again the figure of merit: as it approaches zero, $g_{\text {out }}^{(2)}(\tau) \rightarrow g_{\text {in }}^{(2)}(\tau)$ [Eqs. (9) and (11)]. What, however, are quantitatively the conditions under which Bob's filter will act as a two-state system, i.e., exhibit near perfect short-time photon anticorrelation? Short times map to the spectral wings, where unfiltered light from Alice's shot noise is highly detrimental. The front curves of frames (a) and (b) in Fig. 4 correspond to the parameters of frames (c) and (d) in Fig. 3. They show (a)
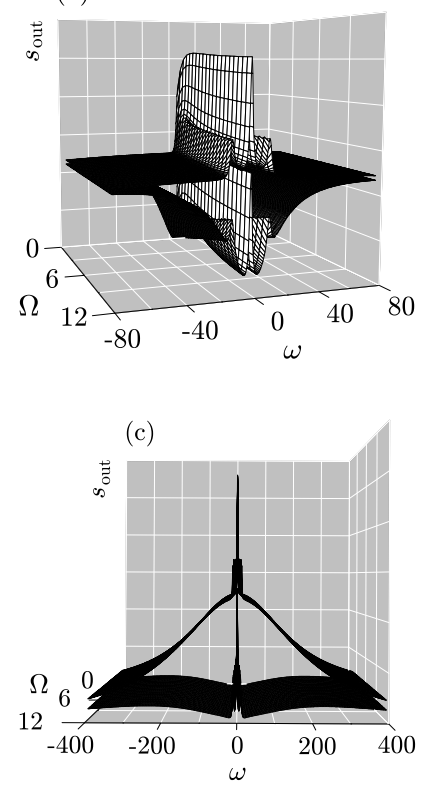

(b)
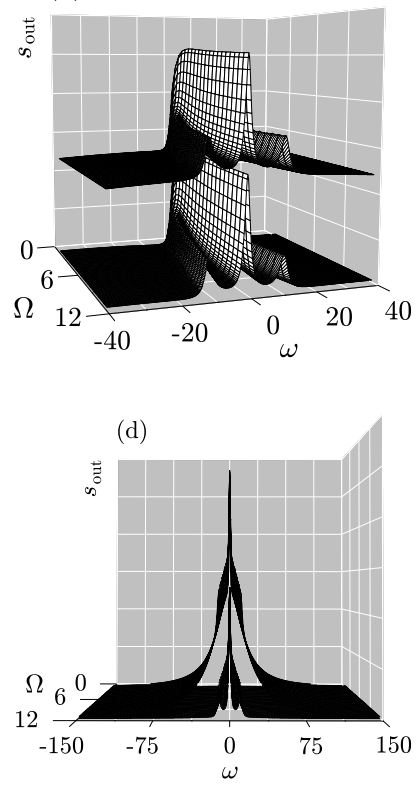

FIG. 3: Teleportation of the Mollow spectrum: $s_{\text {out }}(\omega)$ is plotted for $\left(\gamma_{i}, \gamma_{A}, \gamma_{B}\right) / \gamma_{s}=(0.1,50,100)(\mathrm{a}) ;(0.005,5,1)(\mathrm{b}, \mathrm{c})$; and $(0.005,5,0.1)(\mathrm{d})$. Upper and lower surfaces correspond, respectively, to $0 \mathrm{~dB}$ and $25 \mathrm{~dB}$ line-center squeezing. The Rabi frequency and frequency, $\Omega$ and $\omega$, are in units of $\gamma_{i}$. 

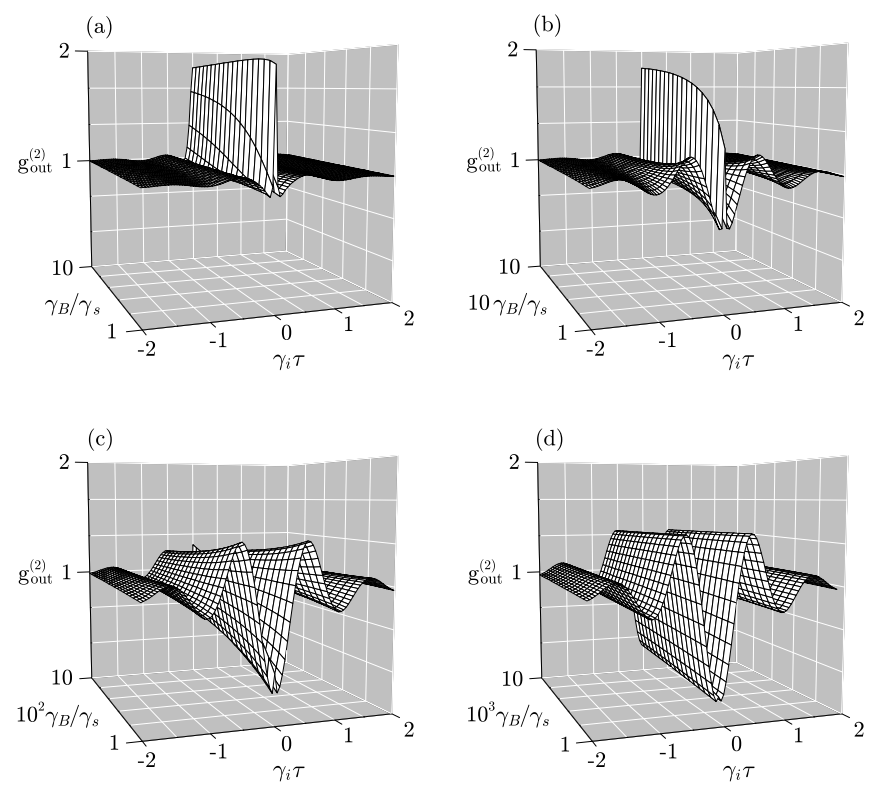

FIG. 4: Teleportation of photon antibunching: $g_{\text {out }}^{(2)}(\tau)$ is plotted for $25 \mathrm{~dB}$ line-center squeezing, $\gamma_{A} / \gamma_{s}=5, \gamma_{B} / \gamma_{i}=20$, and $\gamma_{B} / \gamma_{s}$ varying over four orders of magnitude [(a)-(d)]; Rabi frequency $\Omega / \gamma_{i}=6$.

a prominent spike at zero delay, i.e., bunched rather than antibunched photons. Stronger filtering is needed to teleport photon antibunching [Figs. 4(c) and (d)], though, even then, at $20 \mathrm{~dB}$ line-center squeezing $g_{\text {out }}^{(2)}(0)=0$ is not recovered.

Requirements to achieve $g_{\text {out }}^{(2)}(0) \approx 0$ follow from

$$
g_{\text {out }}^{(2)}(0)=2\left[1-\left(1+f_{s} / f_{\text {in }}\right)^{-2}\right]
$$

[use $A_{s}(0)=g_{\mathrm{in}}^{(1)}(0)=1$ and $g_{\mathrm{in}}^{(2)}(0)=0$ in Eqs. (9) and (11)] and, from Eq. (12), in the limit $\gamma_{B} / \gamma_{s} \rightarrow 0$,

$$
\frac{f_{s}}{f_{\text {in }}}=\frac{\gamma_{B}}{2 \eta \gamma_{i}}\left(\frac{1-\lambda}{1+\lambda}\right)^{2} \frac{\Omega^{2}+2 \gamma_{i}^{2}}{\Omega^{2}}
$$

Thus, a set value of $g_{\text {out }}^{(2)}(0) \ll 1$ calls for

$$
-20 \log \left(\frac{1-\lambda}{1+\lambda}\right)=-10 \log \left(\frac{\Omega^{2}}{\Omega^{2}+2 \gamma_{i}^{2}} \frac{\eta \gamma_{i}}{2 \gamma_{B}} g_{\text {out }}^{(2)}(0)\right)
$$

$\mathrm{dB}$ of line-center squeezing. Then Bob's filter bandwidth is constrained by the need to approximate the $\gamma_{B} / \gamma_{s} \rightarrow 0$ limit [expand the bracket in Eq. (12)]:

$$
\gamma_{B} / \gamma_{s}<\frac{2 \Omega^{2}}{\Omega^{2}+2 \gamma_{i}^{2}} \frac{\eta \gamma_{i}}{2 \gamma_{B}} g_{\text {out }}^{(2)}(0)
$$

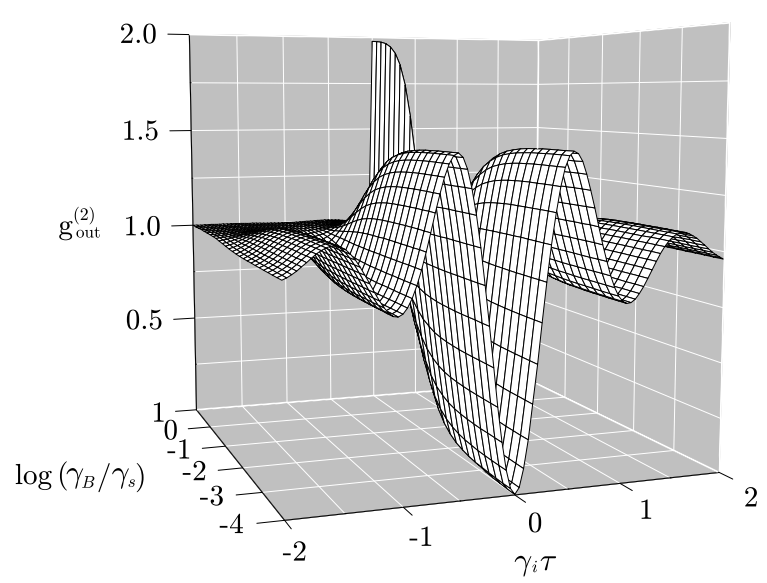

FIG. 5: As in Fig. 4 for $46 \mathrm{~dB}$ line-center squeezing in the limit $\gamma_{A} / \gamma_{s} \gg 1, \gamma_{B} / \gamma_{i} \gg 1$ [Eq. (11)].

Figure 5 illustrates the recovery of near perfect photon anticorrelation, with $g^{(2)}(0) \approx 0.003$ for $\gamma_{B} / \gamma_{s}=10^{-4}$. It demonstrates the central importance of Bob's filter for teleporting an antibunched photon stream. Without it the output has thermal statistics, $g^{(2)}(0)=2$, even though the Mollow triplet is almost perfectly reproduced around the center of the spectrum. The spike at zero delay is caused by a flux of real photons generated by Alice's shot noise: while Wigner noises might formally represent either the vacuum fluctuations of a quantized field or photocurrent shot noise in calculations of quadrature amplitude variances, the two are, in fact, physically distinct - shot noise but not vacuum noise can produce the spike in $g^{(2)}(\tau)$ that Bob's filter must remove.

\section{Acknowledgements}

This work is supported by the Marsden Fund of the RSNZ and HN is supported by grant NPRP 1-7-7-6 of the Qatar National Research Fund.
[1] L. Vaidman, Phys. Rev. A 49, 1473 (1994).

[2] S. L. Braunstein and H. J. Kimble, Phys. Rev. Lett. 80, 869 (1998).

[3] A. Furusawa et al., Science 282, 706 (1998).

[4] W. P. Bowen et al., Phys. Rev. A 67, 032302 (2003).

[5] A. Furusawa and N. Takei, Phys. Rep. 443, 97 (2007).
[6] P. van Loock et al., Phys. Rev. A 62, 022309 (2000).

[7] H. Yonezawa et al., Phys. Rev. Lett. 99110503 (2007).

[8] H. J. Carmichael and H. Nha, "Continuous Variable Teleportation in Stochastic Electrodynamics," in Laser Spectroscopy, eds. P. Hannaford, A. Sidorov, H. Bachor, and K. Baldwin (World Scientific, Singapore, 2003) pp. 324- 
333.

[9] H. J. Carmichael, "Continuous Variable Teleportation of Quantum Fields," in Quantum Information Science, eds. C. Soo and W.-M. Zhang (World Scientific, Singapore, 2005) pp. 11-25.

[10] The formal operators $\hat{I}$ and $\hat{I}^{\dagger}$ commute; $\hat{I}$ is not a legitimate field operator. The notation simply helps us track correlations. Essentially we follow the all optical teleportation scheme of Ralph [11], except that Ralph introduces a genuine quantum field in place of Alice's photocurrent, then, after amplifying it, neglects precisely those terms that make it a quantum field.

[11] T. C. Ralph, Opt. Lett. 24, 348 (1999). 